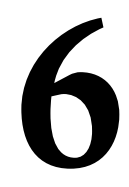

\title{
PERCEPCIÓN DE LOS ESTUDIANTES SOBRE EL VALOR DE LAS TIC EN SUS ESTRATEGIAS DE APRENDIZAJE Y SU RELACIÓN CON EL RENDIMIENTO
}

\section{(STUDENT PERCEPTION OF THE VALUE OF ICT'S IN THEIR LEARNING STRATEGIES AND THEIR RELATION TO PERFORMANCE)}

Ana García-Valcárcel Muñoz-Repiso

Francisco Javier Tejedor Tejedor

Universidad de Salamanca

DOI: 10.5944/educXX1.19035

\section{Cómo referenciar este artículo/How to reference this article:}

García-Valcárcel, A. y Tejedor Tejedor, F. J. (2017). Percepción de los estudiantes sobre el valor de las TIC en sus estrategias de aprendizaje y su relación con el rendimiento. Educación XX1, 20(2), 137-159, doi: 10.5944/educXX1.19035

García-Valcárcel, A. \& Tejedor Tejedor, F. J. (2017). Student perception of the value of ICT's in their learning strategies and their relation to performance. Educación XX1, 20(2), 137-159, doi: 10.5944/educXX1.19035

\section{RESUMEN}

Actualmente la investigación educativa se centra en la búsqueda de evidencias que pongan de manifiesto la utilidad de las TIC (Tecnologías de la Información y Comunicación) para la mejora del rendimiento académico de los universitarios. En esta línea, nuestra investigación pretende conocer el potencial de las TIC para el desarrollo de estrategias de aprendizaje relevantes de cara a mejorar el desempeño de las tareas académicas y el rendimiento. Llevamos a cabo para ello el estudio de la variable "Valoración de las TIC para el desarrollo de estrategias de aprendizaje». Profundizamos en el análisis de esta variable estableciendo, a partir de los resultados del análisis factorial, dos tipos diferenciados de estrategias de aprendizaje (de pensamiento y procedimentales). El diseño incluye el análisis de las diferencias en las valoraciones de las TIC entre alumnos con distinto nivel de rendimiento académico. Como novedad incorporamos la representación gráfica de esas diferencias mediante las curvas ROC y el análisis de la similitud de resultados entre los valores AUC y los estadísticos no paramétricos U y W. Entre las conclusiones destacar que los alumnos de mayor éxito académico reconocen en las TIC un mayor potencial de apoyo en sus estrategias de aprendizaje. Las mayores diferencias entre alumnos (consideran- 
do las notas reales obtenidas), las podemos encontrar en la valoración que hacen de las TIC para mejorar la elaboración de trabajos, la organización de su actividad académica, las tareas de repaso, el trabajo con compañeros y la búsqueda de recursos.

\section{PALABRAS CLAVE}

Estrategia de aprendizaje; Rendimiento; Tecnologías de la Información y Comunicación; Estadística no paramétrica; Estudiante universitario.

\section{ABSTRACT}

Currently, educational research is focused on searching for evidence to show the usefulness of ICT'S (Information and Communication Technologies) for improved academic performance at university. So, our research aims to discover the potential of ICT's as a tool to develop learning strategies for improving the performance of academic tasks. For this, we studied the variable "Assessment of ICT's for the development of learning strategies». We delved into the analysis of this variable, considering the results of the factor analysis, which allow us to distinguish two types of strategies (thinking and procedural). The design includes the analysis of differences in ICT assessments among students with different levels of academic achievement. A new feature was the incorporation of the graphical representation of these differences using COR curves and analysis of the similarity between the AUC values and the non-parametric statistics $U$ and $W$. The conclusions highlight that dependent variables show higher values in the group of students of higher performance, although all students make a positive assessment of technologies as tools which facilitate finding resources and fulfillment of academic tasks.

\section{KEYWORDS}

Learning Strategy; Achievement; Information and Communication Technologies; Nonparametric Statistical data; Undergraduate Student.

\section{INTRODUCCIÓN}

Son varias las causas que diferencian a los buenos estudiantes de los que no lo son tanto: inteligencia, personalidad, conocimientos previos, motivación, cantidad y calidad de las estrategias que los alumnos ponen en juego cuando aprenden, etc. Las estrategias de aprendizaje están directamente relacionadas con la calidad del aprendizaje del estudiante, y su análisis permite identificar y diagnosticar las causas del bajo rendimiento y mejorar el aprendizaje (Beltrán, 2003; Muñoz, 2005). 
Que los alumnos pongan en marcha determinadas estrategias de aprendizaje no es algo que surge espontáneamente, requiere una práctica intencionada. Por este motivo han surgido propuestas pedagógicas que bajo el titulo de enseñar a aprender, aprender a aprender, intentan formar a los alumnos en estas técnicas de aprendizaje, con la clara intención de favorecer el aprendizaje autónomo y mejorar el rendimiento académico.

La estrategia de aprendizaje se considera como una guía de acciones que hay que seguir; son siempre conscientes e intencionales, dirigidas a un objetivo relacionado con el aprendizaje (Nisbet y Shucksmith, 1987). Las estrategias establecen lo que se necesita para resolver bien la tarea de estudio, seleccionar las técnicas más adecuadas, controlar su aplicación y valorar los resultados (Tuñas, 2007). Las acciones las realiza siempre el alumno, con el objetivo de apoyar y mejorar su aprendizaje. Tienen un alto grado de complejidad, son procedimientos internos fundamentalmente de carácter cognitivo (Martínez y Bonachea, 2011). Pozo (2003) las caracteriza como secuencias integradas de conocimientos o actividades que se eligen con el propósito de facilitar la adquisición, el almacenamiento y/o la utilización de información o conocimientos. El dominio de las estrategias de aprendizaje ayuda al alumno a planificar y organizar sus propias actividades de aprendizaje, requiriendo un cierto grado de conocimiento sobre el propio aprendizaje. Este meta-conocimiento es necesario para que el alumno sea capaz de hacer un uso estratégico de sus habilidades.

Se han identificado, por parte de distintos autores (Herrera, 2014; Noy, 2014; Retamal, 2014; Valle et al., 1999; Weinstein y Mayer, 1985), cinco tipos de estrategias generales en el entorno académico. Las tres primeras son estrategias cognitivas que ayudan al alumno a elaborar y organizar los contenidos para que resulte más fácil el aprendizaje (procesar la información), la cuarta está destinada a controlar la actividad mental del alumno para dirigir el aprendizaje (estrategia metacognitiva) y la quinta sirve de apoyo al aprendizaje para que este se produzca en las mejores condiciones posibles.

1. Estrategias de ensayo. Son aquellas que implican la repetición activa de los contenidos (diciendo, escribiendo) o centrándose en partes claves de los mismos. Se fundamentan en un aprendizaje asociativo basado en la práctica reiterada.

2. Estrategias de elaboración. Implican hacer conexiones entre lo nuevo y lo familiar. Inicialmente, son útiles para el aprendizaje conceptual pudiendo ser posteriormente el germen de aprendizajes más consolidados. 
3. Estrategias de organización. Agrupan la información para que sea más fácil recordarla. Implican estructurar el contenido de aprendizaje, dividiéndolo en partes e identificando relaciones y jerarquías (clasificación, jerarquización).

4. Estrategias de control de la comprensión o metacognitivas. Implican ser consciente de lo que se está tratando de lograr, seguir la pista de las estrategias que se usan y del éxito logrado con ellas y adaptar la conducta en concordancia. Entre las estrategias metacognitivas están: la planificación (los alumnos dirigen y controlan su conducta), la regulación (capacidad del alumno para seguir el plan trazado y comprobar su eficacia) y la evaluación (verificar los resultados del proceso de aprendizaje).

5. Estrategias de apoyo o afectivas. La misión fundamental de estas estrategias es conseguir la eficacia del aprendizaje mejorando las condiciones en las que se produce. Podrían calificarse como instrumentales e incluyen: establecer y mantener la motivación, enfocar la atención, mantener la concentración, manejar la ansiedad, disponer de los recursos necesarios, saber trabajar con otros, etc.

Las estrategias cognitivas son procesos que nos permiten comprender, fijar, elaborar y reestructurar la información; promueven un aprendizaje autónomo, independiente, realizado de manera que las riendas y el control del aprendizaje vayan pasando de las manos del profesor a las de los alumnos, fomentando la capacidad de planificar, regular y evaluar su propio aprendizaje, lo que nos sitúa en el ámbito de lo metacognitivo. Para Rinaudo, Chiercher y Donolo (2003) las estrategias cognitivas implican pensamientos o comportamientos que ayudan a adquirir información y a integrarla al conocimiento ya existente, incluyendo estrategias relacionadas con la atención y con los procesos de codificación (carácter superficial) y estrategias de elaboración y organización, que suponen procesamientos más profundos y pensamiento crítico. Por su parte, las estrategias metacognitivas, como ya se ha apuntado, implican la activación de aspectos superiores del conocimiento relacionadas con el planeamiento, la regulación y el control. Tanto las estrategias cognitivas como las metacognitivas pueden entenderse como estrategias de pensamiento, en el sentido apuntado por Beltrán:

"Nos estamos refiriendo, por tanto, a las actividades $u$ operaciones mentales que el estudiante puede llevar a cabo para facilitar y mejorar la realización de la tarea, cualquiera que sea el ámbito o el contenido del aprendizaje. Las estrategias de aprendizaje, asi entendidas, no son otra cosa que las operaciones que realiza el pensamiento cuando ha de enfrentarse a la tarea del aprendizaje. Podemos imaginarlas como las gran- 
des herramientas del pensamiento puestas en marcha por el estudiante cuando este tiene que comprender un texto, adquirir conocimientos o resolver problemas.» (Beltrán, 2003, 57).

Estas estrategias de pensamiento pueden verse favorecidas o apoyadas por las estrategias de "manejo de recursos o de apoyo», citadas por autores como Ruiz (1999) y Portillo (2011), las cuales incluyen el ambiente de estudio, la regulación del esfuerzo, el aprendizaje con pares y la búsqueda de ayuda, equiparándolas con las «técnicas de trabajo intelectual».

El alumno deberá escoger, de entre su repertorio, la estrategia de aprendizaje más adecuada en función de los siguientes factores (Tuñas, 2007):

a) Los contenidos de aprendizaje, tipo y cantidad;

b) Los conocimientos previos que posea sobre el contenido de aprendizaje;

c) Las condiciones de aprendizaje (tiempo disponible, motivación, condiciones personales del alumno...) y

d) El tipo de evaluación al que va a ser sometido.

Las estrategias que el alumno elija pueden verse favorecidas por el uso de las TIC, por lo que resulta interesante analizar en qué medida los alumnos las valoran, hasta qué punto aprecian las TIC como potenciadoras de su aprendizaje.

En la actualidad el manejo de la inabarcable información a disposición del alumno le exige saber acceder, buscar y, sobre todo, seleccionar la información que puede ayudarle en sus actividades de aprendizaje, así como su estructuración, síntesis, presentación, etc. El dominio de estas habilidades supone la adquisición por parte del alumno de competencias informacionales específicas relacionadas con el uso de las TIC (De Pablos, 2010; García-Valcárcel, 2009; García-Valcárcel y Arras, 2010; Rodríguez, Martínez y Olmos, 2013).

El estudio ImpaCT2 (BECTA, 2002) pone de manifiesto que la mayor implicación del alumno en el proceso de aprendizaje debido al uso de las TIC, mejora las oportunidades para la reflexión, el análisis, la resolución de problemas, el pensamiento crítico, así como el desarrollo de habilidades de comunicación, de colaboración, de aprendizaje independiente y de trabajo en grupo. 


\section{MÉTODO}

\section{Objetivos}

En nuestra investigación pretendemos conocer la opinión del alumnado sobre el potencial de ayuda que reconocen a las TIC con respecto a determinadas estrategias de aprendizaje relevantes para la mejora de las tareas académicas y, por extensión, el rendimiento, lo que llevaremos a cabo con el estudio de las variable «Valoración de las TIC en las estrategias de aprendizaje» (EVATIC). Profundizaremos en el análisis de esta variable estableciendo, a partir de los resultados del análisis factorial, dos tipos diferenciados de estrategias de aprendizaje (de pensamiento y procedimentales). El estudio se completa con el análisis de cada una de las estrategias seleccionadas.

Este objetivo básico se completa contrastando si hay diferencias en las valoraciones de las TIC entre alumnos con distinto nivel de rendimiento académico (alto versus normal), a partir de las calificaciones reales obtenidas por los alumnos.

Asimismo pretendemos realizar los análisis de diferencias entre submuestras, en términos de representación gráfica, a través de las curvas ROC, sometiendo a contraste la equivalencia de los valores AUC (áreas bajo la curva) con los estadísticos no paramétricos U de Mann-Withney y W de Wilconxon.

\section{Variables}

De acuerdo a los objetivos fijados, las variables consideradas en el estudio son las siguientes:

1. Cada uno de los ítems de la escala puede ser considerado como variable aislada. El conjunto de ítems de la escala conforman la variable «Valoración de las TIC en las estrategias de aprendizaje» (EVATIC) definida como la media de la suma de puntuaciones otorgadas por los sujetos al conjunto de ítems de la escala. Se genera una variable de rango de 0 a 4 .

2. A partir de los resultados del análisis factorial creamos dos nuevas variables (que toman respectivamente el nombre de cada uno de los dos factores resultantes retenidos), obteniendo para cada sujeto de la muestra su puntuación media en cada uno de los conjuntos de variables que saturan en cada factor. Así, obtenemos para cada 
sujeto su puntuación en la variable «estrategias de pensamiento» (estra-pensamiento) y en la variable «estrategias procedimentales» (estra-procedimentales).

3. Notas reales. El dato se obtiene a partir de las actas oficiales de calificaciones de los alumnos en la asignatura que ha sido desarrollada en un entorno TIC. Genera una variable de rango 0 a 10.

\section{Muestra}

El estudio se lleva a cabo en la Universidad de Salamanca. La población teórica de referencia queda definida por los grupos de clase, conjunto de profesores y alumnos que están implicados en procesos de innovación didáctica, incorporando el uso intensivo de las TIC en el desarrollo de la asignatura. Para ello se analizan los proyectos de innovación con reconocimiento oficial de la Universidad de Salamanca en el curso objeto de estudio. Se establece como elemento muestral el «grupo clase» de los profesores que aceptan participar, quedando constituida la muestra por 20 profesores y 860 alumnos de los que disponíamos de datos en todas las variables consideradas.

\section{Instrumentos para evaluar el uso de estrategias de aprendizaje}

Se han llevado a cabo diversos estudios sobre las estrategias de aprendizaje que utilizan los estudiantes universitarios. Weinstein, Schulte y Valenzuela (1995) realizaron una adaptación del LASSI a los estudiantes venezolanos creando el Inventario de Estrategias de Estudio y Aprendizaje (IEEA) con diez escalas para evaluar dichas estrategias. López-Aguado (2010) analizó las estrategias en relación con el trabajo autónomo. Muchos de los estudios realizados en España han aplicado la escala ACRA (Román y Gallego, 1994). Entre los más interesantes cabe citar trabajos que han intentado encontrar relaciones entre las técnicas de aprendizaje y el rendimiento académico, utilizando en algunos casos versiones reducidas de la misma escala (De la Fuente y Justicia, 2003; Justicia, Archilla y De la Fuente, 1998).

A partir de la estructura de esta versión y basándonos en los trabajos anteriormente comentados de Rinaudo, Chiecher y Donolo (2003), elaboramos la «Escala de valoración de las TIC en el desarrollo de estrategias de aprendizaje» (EVATIC-DEA), de 9 ítems (ver Tabla 3), y que ha sido el instrumento utilizado en la investigación que ahora presentamos. Nuestra intención con la utilización de esta escala es: 1) Conocer la valoración que 
los alumnos realizan de las TIC en relación a las estrategias que utilizan en su proceso de enseñanza-aprendizaje y 2) Constatar si hay diferencias en las valoraciones entre alumnos de distinto nivel de rendimiento.

Se establecieron para cada ítem cinco categorías de respuesta: Desde «muy en desacuerdo» a «muy de acuerdo», puntuadas de 0 a 4 puntos. El análisis de la fiabilidad de la escala nos proporciona un valor a de Cronbach de 0,869 , lo que dada la longitud de la escala (9 ítems), nos permite considerarla como óptima.

El análisis factorial realizado, con rotación varimax, nos proporciona la información que presentamos en las Tablas 1 y 2. En la primera de ellas vemos la estructura factorial resultante y las saturaciones de cada variable (estrategias) en cada factor. El criterio de retener los factores con valores $\lambda \geq 1$ determina el reconocimiento de dos factores que explican el 61,5\% de la varianza. En la matriz de componentes rotados (Tabla 2) vemos la saturación de cada variable en cada uno de los factores retenidos. Su observación nos permite determinar con claridad la tipología y correspondiente denominación de cada uno de los factores:

- En el primer factor las saturaciones más elevadas (superiores a 0,50) corresponden a las variables (estrategias) número 6, 7, 5, 4 y 1 (ver Tabla 1) que se vinculan con las denominadas «estrategias de pensamiento», que agrupan las cognitivas y las metacognitivas (Beltrán, 2003, 59; Gallardo, 2009; Maturano, Soliveres y Macías, 2002).

- En el segundo factor las saturaciones superiores a 0,50 se producen respecto a las cuatro estrategias restantes $(9,2,8$ y 3$)$ que se vinculan con las estrategias denominadas «de organización, procedimentales o instrumentales».

Esta estructura, simplificada dado el número de estrategias incluidas en la escala, puede considerarse coherente con los datos presentados en los apartados anteriores (Portillo, 2011; Rinaudo, Chiercher y Donolo, 2003; Ruiz, 1999) por lo que consideramos que su utilización puede reportarnos información fiable y, desde una perspectiva estructural y teórica, válida. 
Tabla 1

Análisis factorial de la escala «EVATIC-DEA». Factores obtenidos y varianza explicada

\begin{tabular}{|c|c|c|c|c|c|c|c|c|c|}
\hline \multirow[t]{2}{*}{ Factor } & \multicolumn{3}{|c|}{ Autovalores iniciales } & \multicolumn{3}{|c|}{$\begin{array}{c}\text { Sumas de las saturaciones } \\
\text { al cuadrado de la extrac- } \\
\text { ción }\end{array}$} & \multicolumn{3}{|c|}{$\begin{array}{l}\text { Suma de las saturaciones } \\
\text { al cuadrado de la rotación }\end{array}$} \\
\hline & Total & $\begin{array}{c}\% \text { de la } \\
\text { varianza }\end{array}$ & $\begin{array}{c}\% \text { acu- } \\
\text { mulado }\end{array}$ & Total & $\begin{array}{c}\% \text { de la } \\
\text { varianza }\end{array}$ & $\begin{array}{l}\% \text { acu- } \\
\text { mulado }\end{array}$ & Total & $\begin{array}{c}\% \text { de la } \\
\text { varianza }\end{array}$ & $\begin{array}{l}\% \text { acu- } \\
\text { mulado }\end{array}$ \\
\hline 1 & 4,425 & 49,171 & 49,171 & 4,425 & 49,171 & 49,171 & 2,928 & 32,535 & 32,535 \\
\hline 2 & 1,113 & 12,362 & 61,533 & 1,113 & 12,362 & 61,533 & 2,610 & 28,998 & 61,533 \\
\hline 3 & 0,835 & 9,279 & 70,812 & & & & & & \\
\hline 4 & 0,650 & 7,219 & 78,031 & & & & & & \\
\hline 5 & 0,482 & 5,360 & 83,391 & & & & & & \\
\hline 6 & 0,464 & 5,153 & 88,544 & & & & & & \\
\hline 7 & 0,392 & 4,356 & 92,900 & & & & & & \\
\hline 8 & 0,328 & 3,647 & 96,547 & & & & & & \\
\hline 9 & 0,311 & 3,453 & 100,000 & & & & & & \\
\hline
\end{tabular}

Tabla 2

Análisis factorial de la escala. Matriz de componentes rotados

\begin{tabular}{l|c|c}
\hline \multirow{2}{*}{} & \multicolumn{2}{|c}{ Factor } \\
\cline { 2 - 3 } & $\mathbf{1}$ & $\mathbf{2}$ \\
\hline Estrategia 1 & 0,559 & $\mathbf{0 , 4 6 9}$ \\
Estrategia 2 & 0,273 & 0,818 \\
Estrategia 3 & 0,493 & 0,566 \\
Estrategia 4 & 0,663 & 0,291 \\
Estrategia 5 & 0,683 & 0,327 \\
Estrategia 6 & 0,835 & 0,087 \\
Estrategia 7 & 0,783 & 0,186 \\
Estrategia 8 & 0,275 & 0,653 \\
Estrategia 9 & 0,082 & 0,861 \\
\hline
\end{tabular}

\section{Análisis de datos}

Análisis de la valoración de las TIC para el desarrollo de las estrategias de aprendizaje

En la Tabla 3 presentamos los porcentajes de las frecuencias de respuesta para cada una de las categorías de los ítems de la escala. Añadimos la columna que informa del porcentaje conjunto de las categorías de respuesta 
3 y 4 («de acuerdo» $\mathrm{y}$ «muy de acuerdo»), entendiendo que este dato informa de las valoraciones positivas.

Tabla 3

Valoración de los alumnos sobre las TIC en las estrategias de aprendizaje

\section{Las TIC y las estrategias de aprendizaje}

Considero que el uso de las TIC:

Porcentajes de respuesta por categorías $(*)$

1. Favorece mis tareas de repaso de
aprendizajes
2. Mejora la elaboración de trabajos
académicos

3. Me permite organizar mejor mi trabajo académico

4. Me permite desarrollar mejor un pensamiento crítico

5. Me posibilita una mejor organización cognitiva

6. Me permite optimizar el manejo del tiempo que dedico al estudio

7. Favorece la regulación del esfuerzo que dedico a las tareas académicas.

8. Mejora las posibilidades de trabajar con otros compañeros.

9. Favorece la búsqueda de recursos que me ayudan en las tareas académicas.

(*) 0 = Muy en desacuerdo; 1 = En desacuerdo; 2 = Indiferente;

3 = De acuerdo; 4 = Muy de acuerdo

Podemos observar que el porcentaje de respuesta más alto en el conjunto de categorías más favorables $(3+4)$ se produce para la estrategia 9 ( «favorece la búsqueda de recursos que me ayudan en las tareas académicas»). Esto sería lo que los estudiantes consideran como aportación más valiosa de las TIC para sus tareas de aprendizaje. La ordenación de las valoraciones positivas del conjunto de las estrategias resulta ser:

- favorecen la búsqueda de recursos para las tareas académicas

- mejoran la elaboración de los trabajos académicos 
- mejoran la posibilidad de trabajo con otros compañeros

- favorecen mis tareas de repaso de aprendizajes

- me permiten organizar mejor mi trabajo

- favorecen la regulación del esfuerzo

- me posibilitan una mejor organización cognitiva

La valoración menos positiva (no llegan al $50 \%$ los alumnos que claramente se manifiestan de acuerdo con el ítem) se refiere a las estrategias:

- me permiten optimizar el manejo del tiempo

- las TIC me permiten desarrollar mejor un pensamiento crítico

En la Tabla 4 presentamos las medias de la valoración de las TIC (variable EVATIC) realizada por los estudiantes respecto a cada una de las estrategias de aprendizaje, lo que nos permite igualmente confirmar los comentarios realizados, encontrando también una media moderada en el ítem relativo a la regulación del esfuerzo dedicado a las tareas académicas. De este modo, se puede decir que el uso de las TIC en las estrategias de aprendizaje no se asocia a una mejor gestión del tiempo ni sirve al estímulo del pensamiento crítico ni a un menor esfuerzo académico, si bien facilita la realización de las tareas académicas, sobre todo en lo referente a la búsqueda de recursos y a la elaboración de los trabajos.

Las dos variables creadas a partir del análisis factorial tienen (ver Tabla 4) para el conjunto de la muestra unas puntuaciones medias de 2,50 (estrategias de pensamiento) y 3,01 (estrategias procedimentales). Estas puntuaciones muestran que los alumnos reconocen mayor valor a las TIC en relación con las estrategias «procedimentales o instrumentales» (organización y elaboración de trabajos, trabajo con otros, búsqueda de recursos), frente a las estrategias más específicamente «cognitivas» (tareas de repaso, pensamiento crítico, organización cognitiva, manejo del tiempo, regulación del esfuerzo). La correlación entre las puntuaciones de ambas variables es 0,63. La diferencia de medias entre las variables, para muestras correlacionadas, es altamente significativa $(\mathrm{t}=26,38 ; \mathrm{p}=0,000)$.

Diferencias en la valoración de las TIC para el desarrollo de estrategias de aprendizaje en función del rendimiento académico

Para conocer la relación existente entre la valoración que el alumno realiza de las TIC como apoyo a sus estrategias de aprendizaje y el rendimiento académico consideramos la variable «nota real» de los alumnos, con 
recorrido de 0 a 10 puntos, que responde a la calificación real obtenida en la convocatoria de junio en la asignatura que ha demandado un uso significativo de las TIC. Categorizamos dicha variable en dos categorías: rendimiento alto (nota de 7 o superior) y rendimiento normal (nota inferior a 7).

En la Tabla 4 presentamos las medias de cada ítem para las submuestras de alumnos con rendimiento alto (grupo r-alto) y normal (grupo r-normal). En todos los ítems los alumnos del grupo alto tienen medias más altas. En el estudio de la significación de diferencias de medias, tanto en la consideración no paramétrica (z para la U de Mann-Whitney) como en la paramétrica ( $\mathrm{t}$ para muestras independientes), encontramos diferencias altamente significativas en la valoración de las TIC como apoyo para el desarrollo de todas las estrategias de aprendizaje.

El análisis de diferencias por rendimiento para las variables generadas tras el análisis factorial nos proporciona los valores medios que aparecen en la Tabla 4. Observamos que los alumnos del grupo r-alto tienen puntuaciones medias más altas que los del grupo r-normal en las dos variables, lo que nos indica que su valoración de las TIC es más positiva respecto a cualquiera de las macro estrategias estudiadas (de pensamiento y procedimentales). El análisis de las diferencias entre las medias de ambas submuestras resulta altamente significativo para los dos tipos de estrategias.

Tabla 4

Valoración de los alumnos sobre las TIC en las estrategias de aprendizaje. Diferencias por rendimiento

\begin{tabular}{|c|c|c|c|c|c|}
\hline $\begin{array}{c}\text { Estrategias y variables } \\
\text { globales }\end{array}$ & $\begin{array}{l}\text { Media } \\
\text { muestra- } \\
\text { global }\end{array}$ & $\begin{array}{c}\text { Media } \\
\text { grupo } \\
\text { r-normal }\end{array}$ & $\begin{array}{l}\text { Media } \\
\text { grupo } \\
\text { r-alto }\end{array}$ & $\begin{array}{c}\mathbf{z} \\
\text { (U-MW) }\end{array}$ & $\mathbf{T}$ \\
\hline $\begin{array}{l}\text { Favorece mis tareas de } \\
\text { repaso de aprendizajes }\end{array}$ & 2,81 & 2,66 & 2,92 & $-4,40 * *$ & $-4,53 * *$ \\
\hline $\begin{array}{l}\text { 2. Mejora la elaboración } \\
\text { de trabajos académicos }\end{array}$ & 3,10 & 2,86 & 3,27 & $-7,43 * *$ & $-7,31 * *$ \\
\hline $\begin{array}{l}\text { 3. Me permite organizar } \\
\text { mejor mi trabajo aca- } \\
\text { démico }\end{array}$ & 2,79 & 2,61 & 2,93 & $-5,15^{* *}$ & $-5,37 * *$ \\
\hline $\begin{array}{l}\text { 4. Me permite desarrollar } \\
\text { mejor un pensamiento } \\
\text { crítico }\end{array}$ & 2,45 & 2,33 & 2,54 & $-3,36 * *$ & $-3,61 * *$ \\
\hline
\end{tabular}




\begin{tabular}{|c|c|c|c|c|c|}
\hline $\begin{array}{c}\text { Estrategias y variables } \\
\text { globales }\end{array}$ & $\begin{array}{c}\text { Media } \\
\text { muestra- } \\
\text { global }\end{array}$ & $\begin{array}{c}\text { Media } \\
\text { grupo } \\
\text { r-normal }\end{array}$ & $\begin{array}{l}\text { Media } \\
\text { grupo } \\
\text { r-alto }\end{array}$ & $\begin{array}{c}\mathbf{z} \\
(\mathbf{U}-\mathbf{M W})\end{array}$ & $\mathbf{T}$ \\
\hline $\begin{array}{l}\text { 5. Me posibilita una } \\
\text { mejor organización } \\
\text { cognitiva }\end{array}$ & 2,52 & 2,43 & 2,58 & $-2,53 * *$ & $-2,81 * *$ \\
\hline $\begin{array}{l}\text { 6. Me permite optimizar } \\
\text { el manejo del tiempo } \\
\text { que dedico al estudio }\end{array}$ & 2,29 & 2,15 & 2,38 & $-3,30 * *$ & $-3,53 * *$ \\
\hline $\begin{array}{l}\text { 7. Favorece la regula- } \\
\text { ción del esfuerzo que } \\
\text { dedico a las tareas } \\
\text { académicas. }\end{array}$ & 2,44 & 2,31 & 2,53 & $-3,59 * *$ & $-3,57 * *$ \\
\hline $\begin{array}{l}\text { 8. Mejora las posibili- } \\
\text { dades de trabajar con } \\
\text { otros compañeros. }\end{array}$ & 2,89 & 2,72 & 3,01 & $-5,02 * *$ & $-4,62 * *$ \\
\hline $\begin{array}{l}\text { 9. Favorece la búsqueda } \\
\text { de recursos que me } \\
\text { ayudan en las tareas } \\
\text { académicas. }\end{array}$ & 3,28 & 3,14 & 3,38 & $-3,86 * *$ & $-4,17 * *$ \\
\hline Variable «EVATIC» & 2,73 & 2,58 & 2,84 & $-6.61 * *$ & $-6,32 * *$ \\
\hline $\begin{array}{l}\text { Variable «Estrategias pen- } \\
\text { samiento» }\end{array}$ & 2,50 & 2,38 & 2,59 & $-4,87 * *$ & $-4,70 * *$ \\
\hline $\begin{array}{l}\text { Variable «Estrategias pro- } \\
\text { cedimentales» }\end{array}$ & 3,01 & 2,83 & 3,15 & $-7,22 * *$ & $-6,86^{* *}$ \\
\hline
\end{tabular}

(**) Estadístico significativo para $a=0,01$

Análisis gráfico de diferencias entre las submuestras con las curvas ROC

El análisis de las diferencias, en términos de representación gráfica, entre las variables de carácter global puede completarse recurriendo a las curvas ROC (Franco y Vivo, 2007; García-Valcárcel y Tejedor, 2012). Lo hacemos tomando como variables de estudio las tres variables de carácter global que venimos manejando («Evatic», «Estrategias pensamiento; Estrapensa» y «Estrategias procedimentales; Estraproce») y como variable de clasificación o variable de estado la variable rendimiento, con las dos categorías analizadas: alumnos con alto rendimiento y alumnos con rendimiento normal, considerando para el análisis como caso positivo a los alumnos con rendimiento alto. 
Tabla 5

Casos seleccionados en la variable de estado para realizar el análisis de curvas ROC

\begin{tabular}{l|c}
\hline \multicolumn{1}{c|}{ Rendimiento } & $\begin{array}{c}\text { N válido } \\
\text { (según lista) }\end{array}$ \\
\hline Positivo (rendimiento alto) & 497 \\
Negativo (rendimiento normal) & 363 \\
\hline
\end{tabular}

En la Tabla 5 vemos la selección de alumnos «positivos» (la condición de rendimiento alto, de la variable de estado «rendimiento»). El estudio se realiza con 497 datos positivos y 363 datos negativos.

\section{Curva COR}

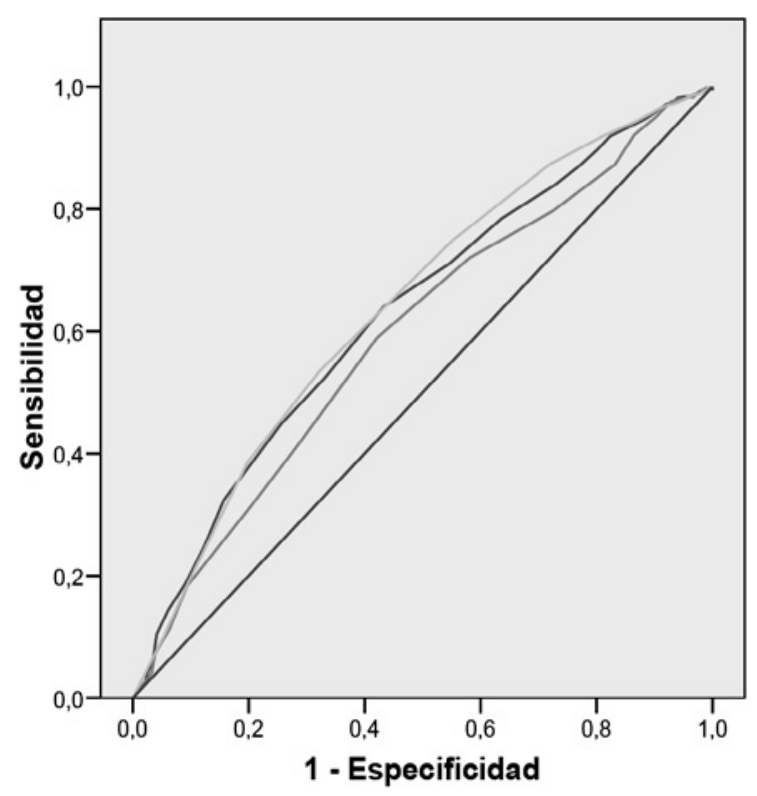

Procedencia de la curva

Evatic

Estrapensa

Estraproce

- Linea de referencia

Figura 1. Curvas ROC - Variable de estado: Alumnos rendimiento alto

En la figura 1 vemos los pares de valores (1-especificidad, sensibilidad) que generan las curvas ROC para cada una de las variables de estudio ( tic», «Estrapensa»y «Estraproce». Observamos que todas las curvas están por encima de la línea diagonal de referencia. Esto ocurre así por el hecho de que las medias en la sub-muestra de alumnos con rendimiento alto (ver Tabla 4) son más altas que las de los alumnos con rendimiento normal en todas las variables de análisis. La gráfica nos permite observar igualmente que todas las áreas bajo las curvas (valores AUC), al estar por encima de la diagonal, representan una proporción superior a 0,50. 
Tabla 6

Área bajo la curva considerando como variable de estado los alumnos de rendimiento alto

\begin{tabular}{l|c|c|c|c|c}
\hline & & & & \multicolumn{2}{|c}{$\begin{array}{c}\text { Intervalo de confianza } \\
\text { Varintótico al 95 \% }\end{array}$} \\
\hline Valoración TIC (Evatic) & 0,631 & 0,019 & 0,000 & 0,594 & 0,669 \\
& curva (AUC) & Error típico análisis & $\begin{array}{c}\text { Significación } \\
\text { asintótica }\end{array}$ & $\begin{array}{c}\text { Límite } \\
\text { superior }\end{array}$ & $\begin{array}{c}\text { Límite } \\
\text { inferior }\end{array}$ \\
\hline Estrategias pensamiento & 0,597 & 0,019 & 0,000 & 0,558 & 0,635 \\
Estrategias & 0,642 & 0,019 & 0,000 & 0,605 & 0,680 \\
procedimentales & & & & & \\
\hline
\end{tabular}

En la Tabla 6 vemos las «áreas bajo la curva» para cada una de las variables. Todos los valores de la columna «área» están por encima y alejados de 0,50, lo que nos indica que el poder de diferenciación de las variables estudiadas respecto a la variable de estado "rendimiento» refleja una diferencia altamente significativa entre las dos sub-muestras para las tres variables, como podemos ver en la columna «significación asintótica» o viendo que entre los límites superior e inferior del intervalo confidencial no se encuentra el valor 0,50 .

La interpretación final, por tanto, se realizaría en los términos siguientes: las variables estudiadas asocian valores más altos con el grupo de alumnos de rendimiento alto, con diferencias significativas inclusive para $a=0,01$.

Similitud entre los análisis de diferencias con curvas ROC y los análisis no paramétricos (U de Mann-Wihtney y W de Wilcoxon).

Pasamos a mostrar cómo el valor del «área bajo la curva» (valor AUC) es equivalente al estadístico U de Man-Withney (prueba no paramétrica para muestras independientes). La equivalencia entre ambos estadísticos se produce en los siguientes términos:

$\mathrm{AUC}=\mathrm{U} /\left(\mathrm{n}_{1} \cdot \mathrm{n}_{2}\right)$

Donde AUC = Valor del área bajo la curva ROC

$\mathrm{U}=$ Valor del estadístico de Mann-Withney

$\mathrm{n}_{1}, \mathrm{n}_{2}=$ Número de sujetos pertenecientes a cada uno de los subgrupos de la variable de estado. 
El valor de AUC es también equivalente a la prueba de lo signos de Wilcoxon, dada la equivalencia que existe entre los estadísticos de esta prueba (W) y el estadístico U de Mann-Withney. La equivalencia entre ambos estadísticos se establece en los siguientes términos:

$$
\mathrm{W}=\left\{\mathrm{n}_{\mathrm{a}}\left(\mathrm{n}_{\mathrm{a}}+1\right) / 2\right\} / 2+\mathrm{U}
$$
de rangos.

Siendo $\mathrm{n}_{\mathrm{a}}$ el tamaño del grupo al que corresponde el menor promedio

Establecida la relación entre los valores de $\mathrm{U}$ y de $\mathrm{W}$ podemos ya relacionar el valor $\mathrm{W}$ con el valor de AUC:

$$
\mathrm{AUC}=\mathrm{U} /\left(\mathrm{n}_{1}, \mathrm{n}_{2}\right)=\left[\mathrm{W}-\left\{\mathrm{n}_{\mathrm{a}}\left(\mathrm{n}_{\mathrm{a}}+1\right) / 2\right\}\right] /\left(\mathrm{n}_{1}, \mathrm{n}_{2}\right)
$$

Verifiquemos, exclusivamente para la variable «EVATIC», el cumplimiento de estas similitudes entre los estadísticos analizados. En la Tabla 7 recogemos los datos necesarios para el cálculo de los estadísticos de contraste U y W.

Tabla 7

Cálculo de rangos necesarios y de los estadísticos $U$ y $W$ (con valores $Z$ y su significación

\begin{tabular}{l|c|c|c|c|c|c|c|c}
\hline Variable & Rendimiento & $\mathbf{N}$ & $\begin{array}{c}\text { Rango } \\
\text { promedio }\end{array}$ & $\begin{array}{c}\text { Suma de } \\
\text { rangos }\end{array}$ & $\mathbf{U}$ & $\mathbf{W}$ & $\mathbf{Z}$ & $\begin{array}{c}\text { Significación } \\
\text { asintótica }\end{array}$ \\
\hline Evatic & Normal & 363 & 365,20 & 132566,00 & 66500 & 132566,00 & $-6,61$ & 0,000 \\
& Alto & 497 & 478,20 & 237664,00 & & & & \\
\hline
\end{tabular}

Si calculamos el valor AUC a partir del correspondiente valor de U tendremos:

$\mathrm{AUC}=\mathrm{U} /\left(\mathrm{n}_{1} \cdot \mathrm{n}_{2}\right)=66500 /(363 \times 497)=0,3686$. El complemento a 1 de dicho valor $(1-0,3686=0,6314)$ vemos que coincide con el valor AUC de la Tabla 6 .

De forma similar comprobamos la relación que existe entre el valor AUC y la W de Wilcoxon:

AUC $=[132566-\{(363 \times 364) / 2\} /(363 \times 497)]=0,3686$. Su complemento a 1 nos ofrecería el valor presentado en la Tabla 6.

\section{DISCUSIÓN Y CONCLUSIÓN}

La interpretación de los datos obtenidos se realizaría en los términos siguientes: las variables dependientes estudiadas (valoración de las TIC por parte de los estudiantes universitarios en relación con las estrategias 
de aprendizaje) asocian valores más elevados con el grupo de alumnos de rendimiento alto, lo que nos indica que los alumnos de mayor éxito académico reconocen en las TIC un mayor potencial de apoyo en sus estrategias de aprendizaje (con diferencias claramente significativas).

Las mayores diferencias entre alumnos con rendimiento alto y normal (considerando las notas reales obtenidas), las podemos encontrar en las valoraciones que hacen de las TIC para mejorar la elaboración de trabajos, la organización de su actividad académica, las tareas de repaso, el trabajo con compañeros y la búsqueda de recursos. En conclusión, si los estudiantes que obtienen mejores calificaciones están manifestando una valoración más positiva de las TIC en sus procesos de aprendizaje, esta debería ser una variable a considerar en los modelos que tratan de explicar el rendimiento académico en la educación superior y en las formas de aprender de los alumnos. Consideramos, por tanto, que los modelos tradicionales que intentan explicar el éxito académico (Baeten, Kyndt, Struyen, y Dochy, 2010; Gargallo et al, 2014; Monroy y Hernández, 2014) deberían incorporar, como variable de estudio, el análisis de la valoración y el uso que hacen los estudiantes de las herramientas tecnológicas para acceder y gestionar la información que va a ser la fuente de su aprendizaje, más cuando en las metodologías centradas en el estudiante, se ofrecen entornos de aprendizaje abiertos y ricos en información, en los que el estudiante debe desarrollar una importante actividad de búsqueda, organización y estructuración de los contenidos de aprendizaje.

Harrington y Parker (2013) apuestan por desarrollar un currículo que capacite a los estudiantes en habilidades de auto-regulación, colaboración y reflexión en contextos de aprendizaje auténticos. De este modo los estudiantes exploran el papel de las TIC en su vida social y en su rol de universitarios, utilizando las tecnologías para resolver problemas y crear oportunidades en su vida cotidiana y familiar. Entre sus conclusiones apuntan, en la misma línea de Kim y Reeves (2007), que el uso de las tecnologías permite ampliar las capacidades de aprendizaje cuando se usan como herramientas cognitivas, soportes para la comunicación y colaboración, plataformas para la publicación de productos elaborados por los propios estudiantes, herramientas cognitivas para el pensamiento y la creación.

Los resultados de esta investigación, coincidentes con las opiniones de diversos autores (Badía y Monereo, 2008; Fandos y González, 2011; Ferro, Martínez y Otero, 2009), confirman sin ninguna duda que el alumno valora con claridad la incidencia de las TIC en el desarrollo de eficaces estrategias de aprendizaje, facilitando las tareas académicas, lo que puede convertirse en una de las claves del éxito académico. Por otra parte, los 
resultados del estudio matizan algunas conclusiones puestas de manifiesto en el estudio ImpaCT2 (BECTA, 2002), el cual afirmaba que la mayor implicación del alumno en el proceso de aprendizaje mejora las oportunidades para la reflexión, el análisis, la resolución de problemas y el pensamiento crítico. En nuestro estudio la reflexión, el análisis y el pensamiento crítico no están especialmente vinculadas al uso de las TIC en opinión de los propios estudiantes universitarios.

La valoración positiva de las TIC en el desarrollo de estrategias de aprendizaje eficaces se encuentra también en consonancia con el estudio de Domínguez (2009) quien afirma que las tecnologías promueven el aprendizaje autónomo, el trabajo colaborativo, el pensamiento creativo, el desarrollo de estrategias de resolución de problemas, el trabajo interactivo, la capacidad de argumentación oral y escrita, la creación de representaciones no lingüísticas de lo que aprenden los alumnos. Todo esto en entornos abiertos (no dirigidos) de aprendizaje, con disponibilidad de herramientas útiles que favorecen una mayor capacidad para investigar, organizar, evaluar y comunicar información, así como la creación de comunidades educativas virtuales.

La consideración de las TIC como herramientas destacables que contribuyen a reforzar los procesos de enseñanza-aprendizaje es la propuesta del «Institute for Human and Machine Cognition» cuyo objetivo de investigación es proporcionar ayuda para construir y compartir modelos de conocimiento basados en mapas conceptuales a través de software específico y el uso de Internet (Cañas at al, 2000). Buzán y Buzán (1999) informan, a partir de sus estudios, que la retención, la comprensión, la creatividad y la capacidad comunicativa de los estudiantes se pueden beneficiar a partir de la asociación de dibujos e imágenes. Interesantes son también las propuestas de Johnson y Johnson (1999) en relación con el aprendizaje colaborativo y los estudios de Esteban y Zapata (2008) que analizan las estrategias de aprendizaje que ponen en juego los alumnos en el marco de e-learning.

Finalmente destacar que teniendo en cuenta la crítica realizada por relevantes autores (Kirkwood y Price, 2013; Reeves, 2011), desde un paradigma fundamentalmente interpretativo, a las investigaciones que pretenden evaluar el impacto de las TIC en el aprendizaje a través de una evaluación pretendidamente objetiva de las prácticas tecnológicas de enseñanza en vez de atender a dimensiones más pedagógicas relacionadas con el tipo de tareas, la coherencia de la evaluación, etc.; nuestro trabajo se ha focalizado, no tanto en el uso de determinadas herramientas tecnológicas por parte de los alumnos sino en su incidencia en las estrategias de aprendizaje, las cuales deberían ser las verdaderas beneficiadas del uso de los 
nuevos dispositivos tecnológicos. Las tecnologías deberían permitir a los estudiantes, no solo "hacer las cosas mejor», sino "hacer mejores cosas» (Reilly, 2005), en el sentido de enriquecer los procesos de aprendizaje consiguiendo una mayor profundidad y significatividad en los conocimientos adquiridos y unas mejores actitudes hacia el estudio, potenciando una mayor autonomía y autogestión de los procesos de aprendizaje, desarrollando destrezas cognitivas y metacognitivas, además de instrumentales, que les permitan seguir aprendiendo de forma permanente. 


\section{REFERENCIAS BIBLIOGRÁFICAS}

Baeten, M., Kyndt, E., Struyen, K. \&Dochy, F. (2010). Using student-centred learning environments to stimulate deep approaches to learning: factors encouraging or discouragingtheir effectiveness. Educational Research Review, 5, 243-260.

Badia, A. y Monereo, C. (2008). La enseñanza y el aprendizaje de estrategias de aprendizaje en entornos virtuales. En C. Coll y C. Monereo (Eds.) Psicología de la educación virtual. Aprender y enseñar con las TIC (pp. 348-367). Madrid: Morata.

Beltrán, J. (2003). Estrategias de aprendizaje. Revista de Educación, 332, 55-73.

British Educational Communications and Technology Agency (BECTA) (2002). ImpaCT2: the impact of information and communication technologies on pupil learning and attainment. Recuperado de http://dera.ioe.ac.uk/1572/

Buzán, T y Buzán, B. (1999). El libro de los mapas mentales: Cómo utilizar al máximo las capacidades de la mente. Barcelona: Urano.

Cañas, A. J., Ford, K. M., Coffey, J., Reichherzer, T., Carff, R. Shamma, G. y Breedy, M. (2000). Herramientas para construir y compartir modelos de conocimientos basados en mapas. Revista de Informática Educativa, 13(2), 145-158.

De la Fuente, J. y Justicia, F. (2003). Escala de estrategias de aprendizaje ACRA-abreviada para alumnos universitarios. Revista Electrónica de Investigación Psicoeducativa y Psicopedagógica, 1(2), 139-58.

De Pablos, J. (2010). Universidad y sociedad del conocimiento. Las competencias informacionales y digitales. Revista de Universidad y Sociedad del Conocimiento (RUSC), 7(2), 6-15. Recuperado de https://goo.gl/DxUOAo.
Domínguez, E. (2009). Las TIC como apoyo al desarrollo de los procesos de pensamiento y la construcción activa de conocimientos. Revista del Instituto de Estudios de Educación, 10, 146-155.

Esteban, M. y Zapata, M. (2008). Estrategias de aprendizaje y eLearning. Un apunte para la fundamentación del diseño educativo en los entornos virtuales de aprendizaje. Consideraciones para la reflexión y el debate. Introducción al estudio de las estrategias y estilos de aprendizaje. RED. Revista de Educación a Distancia, 19. Recuperado de https://goo.gl/2pctjM

Fandos, M. y González, A. P. (2006). Estrategias de Aprendizaje ante las nuevas posibilidades educativas de las TIC. Recuperado de https://goo.gl/3OBDQc.

Ferro, C., Martínez, A. I., Otero, M. C. (2009). Ventajas del uso de las TICs en el proceso de enseñanza-aprendizaje desde la óptica de los docentes universitarios españoles. EDUTEC, Revista Electrónica de Tecnología Educativa. 29, 1-11. Recuperado de https://goo.gl/ nqsL8c

Franco, M. y Vivo, J. M. (2007). Análisis de curvas ROC. Principios y aplicaciones. Madrid: La Muralla.

Gallardo, B. (2009). Estrategias de aprendizaje. Estado de la cuestión. Propuestas para la intervención educativa. Teoría de la Educación. Revista Interuniversitaria, 7, 53-75.

García-Valcárcel, A. (coord.) (2009). La incorporación de las TIC en la docencia universitaria: recursos para la formación del profesorado. Barcelona: Davinci. 
García-Valcárcel, A. y Arras, A. M. (coord.) (2010). Competencias en TIC y rendimiento académico en la Universidad: diferencias por género. México D. F.: Pearson International.

García-Valcárcel, A. \& Tejedor, F. J. (2012). The incorporation of ICT in higher education. The contribution of the ROC curves in the graphic visualization on difference the analysis of the variables. British Journal of Educational Technology, 43(6), 901-919.

Gargallo, B., Morera, I., Iborra, S., Climent, M. J., Navalón, S. y García, E. (2014). Metodología centrada en el aprendizaje. Su impacto en las estrategias de aprendizaje y en el rendimiento académico de los estudiantes universitarios. Revista Española de Pedagogía, 259, 415-435.

Harrington, J. y Parker, J. (2013). Emerging technologies as cognitive tools for authentic learning. British Journal of Educational Technology, 44(4), 607-615.

Herrera, G. (2014). Estrategias de aprendizaje. Monografía. Recuperado de https://goo.gl/3G4FCg

Johnson, D. W. y Johnson, R. T. (1999). Aprender juntos y solos. Buenos Aires: Aique Grupo Editor.

Justicia, F., Archilla, M. I. y De la Fuente, J. (1998). Factores condicionantes de las estrategias de aprendizaje y del rendimiento académico en alumnos universitarios, a través de las escalas ACRA. Revista de Educación de la Universidad de Granada, 11, 193-209.

Kim, B. \& Reeves, T. C. (2007). Reframing research on learning with technology: In search of the meaning of cognitive tools. Instructional Science, 35, 207-256.
Kirkwood, A. y Price, L. (2013). Examining some assumptions and limitations of research on the effects of emerging technologies for teaching and learning in higher education. British Journal of Educational Technology, 44(4), 536-543.

López-Aguado, M. L. (2010). Diseño y análisis del Cuestionario de Estrategias de Trabajo Autónomo (CETA) para estudiantes universitarios. $R e$ vista de Psicodidáctica, 15(1), 77-99.

Martínez, R. y Bonachea, O. (2011). ¿Estrategias de enseñanza o estrategias de aprendizaje? Recuperado de https:// goo.gl/loJP4K

Maturano, C. I., Soliveres, M. A. y Macías, A. (2002). Estrategias cognitivas y metacognitivas en la comprensión de un texto de ciencias. Enseñanza de las ciencias, 20(3), 415-425.

Monroy, F. y Hernández, F. (2014). Factores que influyen en los enfoques de aprendizaje universitario. Una revisión sistemática. Educación $X X I$, 17(2), 105-124. Doi: 10.5944/educxx1.17.2.11481.

Muñoz, M. T. (2005). Estrategias de aprendizaje en estudiantes universitarias. Recuperado de: https://goo.gl/ fqemjf

Nisbet, J. y Shucksmith, J. (1987). Estrategias de aprendizaje. Madrid: Santillana.

Noy, L. A. (2014). Estrategias de aprendizaje. Recuperado de https://goo.gl/ oWpa6E

Portillo, M. C. (2011). Estrategias de aprendizaje: Aprender a aprender. Recuperado de https://goo.gl/dFlrax

Pozo, J. I. (2003). Adquisición de conocimiento. Madrid: Morata. 
Reeves, T. C. (2011). Can educational research be both rigorous and relevant? Educational Designer, 1(4). Recuperado de https://goo.gl/j34WtI

Reilly, R. (2005). Guest editorial webbased instruction: doing things better and doing better things. IEEE Transactions on Education, 48(4), 565-566. oi:10.1109/TE.2005.859218.

Retamal, G. (2014). Estrategias de aprendizaje. Recuperado de https://goo.gl/ oIcQk4

Rinaudo, M. C., Chiecher, A. y Donolo, D. (2003). Motivación y uso de estrategias en estudiantes universitarios. Su evaluación a partir del Motivated Strategies Learning Questionnaire. Anales de Psicología, 19(1), 107-119.

Rodríguez, M. J., Martínez, F. y Olmos, S. (2013). Evaluación de competencias informacionales en educación secundaria: un modelo causal, Cultura y Educación. Revista de teoría, investigación y práctica, 25(3), 361-374.
Román, J. M. y Gallego, S. (1994). Escala de Estrategias de Aprendizaje ACRA. Madrid: TEA Ediciones.

Ruíz, F. (1999). Trabajo intelectual, información y tecnología digital. Educar, 25, 21-32.

Tuñas, J. (2007). Técnicas-estrategias de aprendizaje: diferencias e incursión en las nuevas tecnologías. Recuperado de https://goo.gl/orZSg0

Valle, A., Barca, A., González, R. y Núñez, J. C. (1999). Las estrategias de aprendizaje. Revisión teórica y conceptual. Revista latinoamericana de Psicología, 31(3), 425-461.

Weinstein, C. E. y Mayer, R. E. (1985). The teaching of learning strategies. En M. C. Wittrock (Ed). Handbook of research on teaching. (V. 3, pp. 315-327). New York: Mac Millan.

Weinstein, C. E., Shulte, A. C. y Valenzuela, R. (1995). Inventario de estrategias de estudio y aprendizaje, IEEA. Clearwater, Florida: H\&H Publishing Company. 


\section{PERFIL ACADÉMICO Y PROFESIONAL DE LOS AUTORES}

Ana García-Valcárcel Muñoz-Repiso. Catedrática de Tecnología de la Educación en Dpto. de Didáctica, Organización y Métodos de Investigación, de la Universidad de Salamanca. Directora del Grupo de Investigación GITE-USAL. Miembro de REUNI+D (Red Universitaria de Investigación e Innovación Educativa). Directora de diversos proyectos de innovación e investigación sobre la integración educativa de las tecnologías digitales, videojuegos educativos y evaluación de las competencias digitales de los estudiantes. Actualmente directora del Máster Oficial «Las TIC en Educación».

Francisco Javier Tejedor Tejedor. Catedrático de Metodología de Investigación Educativa desde 1983. Ha sido coordinador de diversos programas de doctorado, dirigiendo más de 50 tesis doctorales. Ha sido IP en un gran número de proyectos $\mathrm{I}+\mathrm{D}+\mathrm{i}$ en convocatorias nacionales y autonómicas, publicando numerosos artículos sobre temas de evaluación del profesorado y procesos de innovación educativa. Tiene reconocidos 6 sexenios de investigación.

Dirección de los autores: Facultad de Educación Universidad de Salamanca

Paseo de Canalejas, 169

37008 Salamanca

E-mail: anagv@usal.es tejedor@usal.es

Fecha Recepción del Artículo: 07. Noviembre. 2014 Fecha modificación Artículo: 24. Noviembre. 2014 Fecha Aceptación del Artículo: 20. Febrero. 2015 Fecha Revisión para Publicación: 09. Marzo. 2017 
